Textures and Microstructures, Vol. 34, pp. 159-169 Reprints available directly from the publisher Photocopying permitted by license only
(C) 2000 OPA (Overseas Publishers Association) N.V. Published by license under the Gordon and Breach Science Publishers imprint. Printed in Malaysia.

\title{
INITIAL AND RECRYSTALLIZATION TEXTURES OF NICKEL ELECTRODEPOSITS
}

\author{
INSOO KIM* and SAE GWANG LEE
}

\author{
School of Materials and Metallurgical Engineering, Kum Oh National \\ University of Technology, Kumi, Kyung Buk, 730-701, Korea
}

(Received 8 October 1999)

\begin{abstract}
The texture of electrodeposits vary with deposition conditions. Electrodeposits texture is also related to microstructure, surface morphology and mechanical properties. When the electrodeposits annealed, the recrystallization texture may be different from the original deposition texture. The surface morphology, the microstructure and the initial and recrystallization textures of nickel electrodeposits vary with deposition conditions.

The $\langle 100\rangle$ and $\langle 110\rangle$ textures of nickel electrodeposits can be obtained from nickel sulfate, nickel chloride and boric acid baths. The size of surface asperities decreases with increasing current density and decreasing temperature. The $\langle 100\rangle$ and $\langle 110\rangle$ textures of nickel electrodeposits changed to the $\langle 100\rangle$ and $\langle\sqrt{3} 10\rangle$ texture, when recrystallized, respectively.
\end{abstract}

Keywords: Electrodeposits; Inverse pole figure; Surface morphology; Microstructure; Recrystallization texture; Strain energy release maximization model

\section{INTRODUCTION}

The texture, microstructure, surface morphology and mechanical properties of electrodeposits are known to vary with electrolysis conditions, such as bath composition, overpotential, $\mathrm{pH}$, current density, bath temperature, etc. (Rashkov et al., 1972; Lee and Chang, 1974; Ye and Lee, 1981; Lee and Ye, 1981; Ye et al., 1992; Kang et al., 1995; Lee et al., 1995).

Electrodeposits are recrystallized when annealed, as in heavily deformed metals. The development of the recrystallization texture in electrodeposits can be related to the deposition texture.

\footnotetext{
* Corresponding author.
} 
Recently, Lee et al. (1995) and Lee (1995) proposed the strain energy release maximization model, in which the absolute maximum internal stress direction of a deformed or fabricated material becomes parallel to the direction of the minimum elastic modulus of recrystallized grains.

This model can explain the recrystallization of copper electrodeposits (Lee et al., 1995; Lee, 1995; 1996b) and deformation texture (Lee, 1995; 1996b).

In this study, nickel deposits with $\langle 100\rangle$ and $\langle 110\rangle$ textures were obtained from a nickel watt bath, their initial and recrystallization textures, surface morphologies and microstructures were investigated.

\section{EXPERIMENTAL PROCEDURE}

A watt solution was used for nickel plating, which was composed of nickel sulfate, $\mathrm{NiSO}_{4} \cdot 6 \mathrm{H}_{2} \mathrm{O}(270 \mathrm{~g} / \mathrm{l})$, nickel chloride, $\mathrm{NiCl}_{2} \cdot 6 \mathrm{H}_{2} \mathrm{O}$ $(45 \mathrm{~g} / \mathrm{l})$ and boric acid, $\mathrm{H}_{3} \mathrm{BO}_{3}(35 \mathrm{~g} / \mathrm{l})$.

An AISI 304 stainless steel plate was polished with 2000-grit emery paper to reduce the possible influence of the texture of the substrate on the nickel deposit when used as a cathode. The surface of the substrate was cleaned by $\mathrm{HCl}$ and distilled water before the plating.

A high purity nickel plate was used as an anode. The distance between the anode and cathode was $6 \mathrm{~cm}$. The current density ranged from 3.0 to $5.0 \mathrm{~A} / \mathrm{dm}^{2}$ and the bath temperature ranged from $40^{\circ} \mathrm{C}$ to $80^{\circ} \mathrm{C}$. The bath was stirred and controlled to maintain a $\mathrm{pH}$ of 3.0.

The nickel deposits of about $30-50 \mu \mathrm{m}$ in thickness were peeled off from the stainless steel cathode.

The annealing of the nickel deposits was carried out at $580^{\circ} \mathrm{C}$ for $1 \mathrm{~h}$. The texture of the deposits was measured by an X-ray diffractometer and a pole figure device. The cross sectional microstructure and the surface of the electrodeposits were observed under an optical microscope and a scanning electron microscope, respectively.

\section{RESULTS AND DISCUSSION}

Figure 1 shows the surface morphologies of nickel electrodeposits with $\langle 100\rangle$ texture, which is characterized by flat square facets. The size of the flat square facets decreased with increasing current density and decreasing temperature. 
(a)

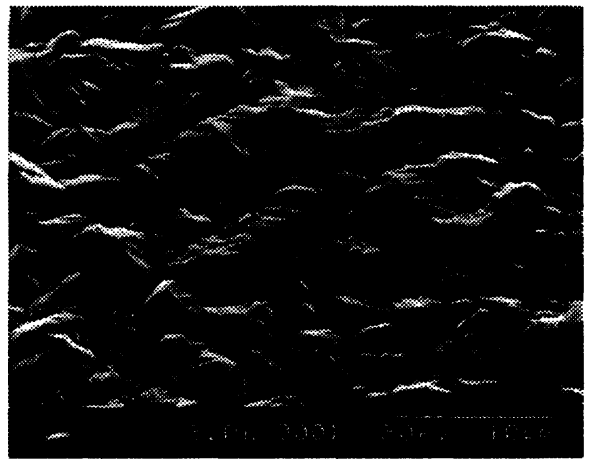

(b)

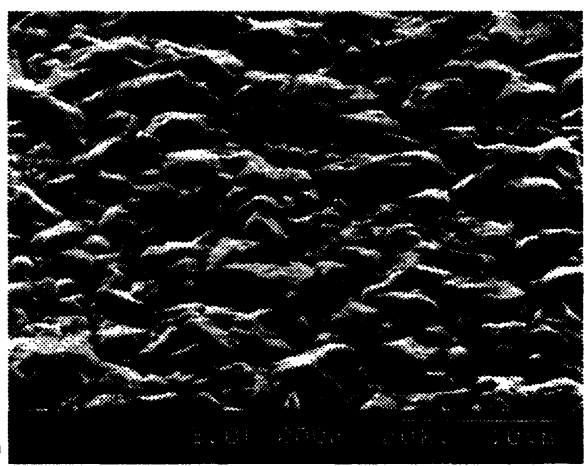

(c)

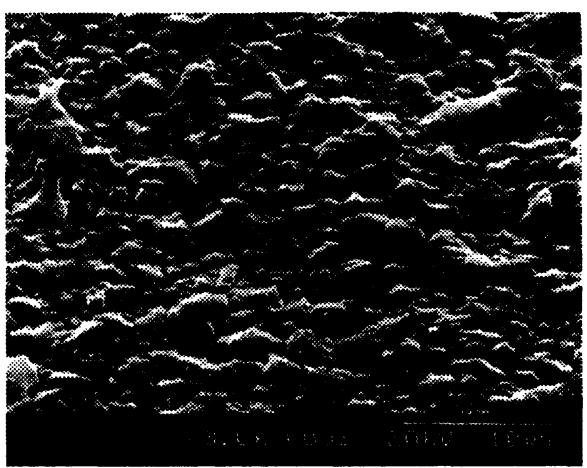

FIGURE 1 Surface morphology of nickel electrodeposits with $\langle 100\rangle$ orientation. Electrolysis conditions - bath temperature: $40^{\circ} \mathrm{C}$, current density: (a) $3 \mathrm{~A} / \mathrm{dm}^{2}$, (b) $4 \mathrm{~A} /$ $\mathrm{dm}^{2}$, (c) $5 \mathrm{~A} / \mathrm{dm}^{2}$.

Figure 2 shows the surface morphologies of nickel electrodeposits with $\langle 110\rangle$ texture, which is characterized by harshness. The size of harshness decreased with increasing current density and decreasing temperature. 
(a)
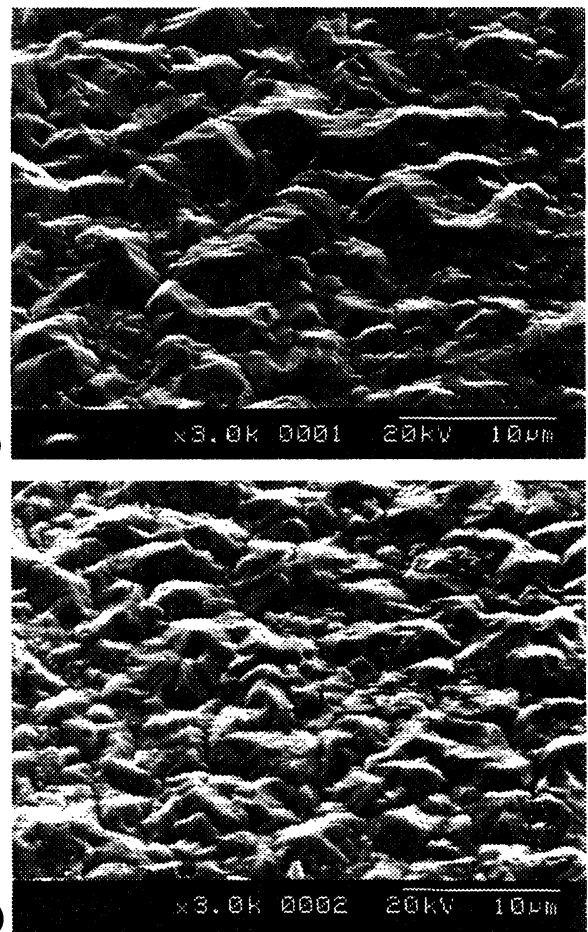

(b)

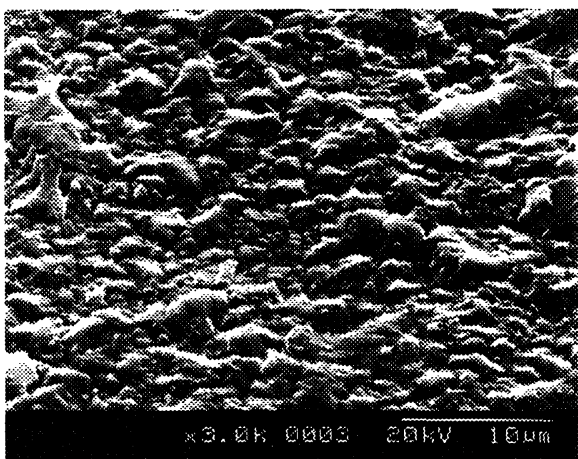

FIGURE 2 Surface morphology of nickel electrodeposits with $\langle 110\rangle$ orientation. Electrolysis conditions - bath temperature: $80^{\circ} \mathrm{C}$, current density: (a) $3 \mathrm{~A} / \mathrm{dm}^{2}$, (b) $4 \mathrm{~A} /$ $\mathrm{dm}^{2}$, (c) $5 \mathrm{~A} / \mathrm{dm}^{2}$.

Figure 3 shows the initial and recrystallized microstructures of the cross section of nickel electrodeposits having $\langle 100\rangle$ texture.

Figure 4 shows the initial and recrystallized microstructures of the cross section of nickel electrodeposits having $\langle 110\rangle$ texture. 

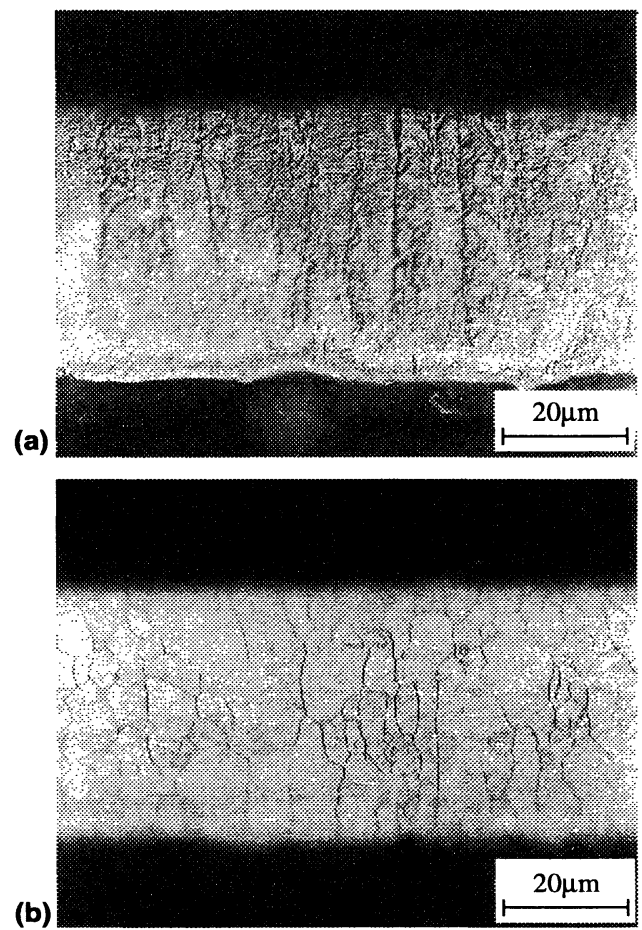

FIGURE 3 Cross sectional microstructure of nickel electrodeposits having $\langle 100\rangle$ texture: (a) as-deposited, (b) recrystallized.

The nickel deposits with the $\langle 100\rangle$ and $\langle 110\rangle$ textures show the welldeveloped columnar structure. The structure of the $\langle 110\rangle$ textured specimen is finer than that of the $\langle 100\rangle$ textured specimen. The traces of twin boundaries make angles of about $35-45^{\circ}$ to the growth direction in the $\langle 100\rangle$ specimen but are parallel to the growth direction in the $\langle 110\rangle$ specimen.

Figure 5 shows the relation between the deposit orientation and $\{111\}$ planes. Figure 5(a) shows that traces of the $\langle 111\rangle$ twin boundaries are at about $35-45^{\circ}$ to the $\langle 100\rangle$ deposit texture. This result is in agreement with the experimental result in Fig. 3(a). Figure 5(b) shows that the traces of the $\langle 111\rangle$ twin boundaries are parallel to the $\langle 110\rangle$ growth direction. This is the reason for the fine columnar boundaries in the deposit with the $\langle 110\rangle$ texture. 
(a)

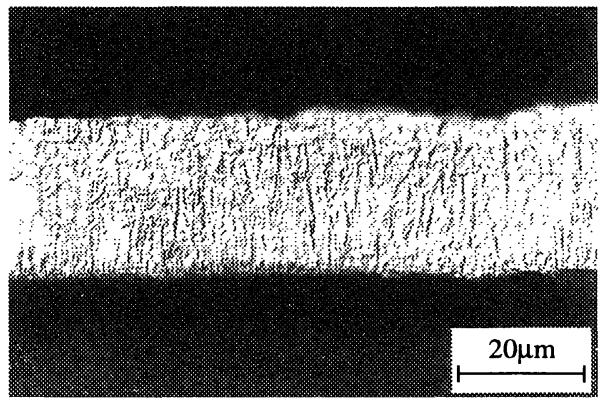

(b)

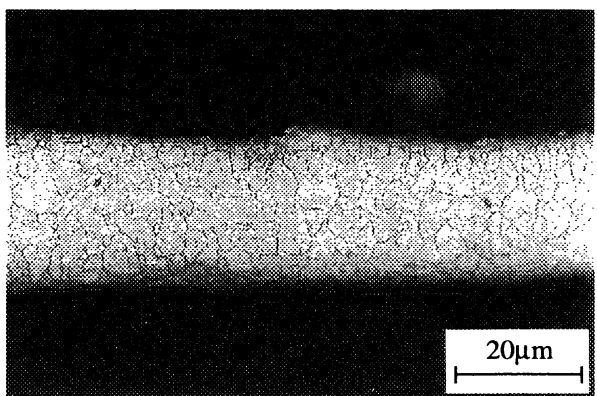

FIGURE 4 Cross sectional microstructure of nickel electrodeposits having $\langle 110\rangle$ texture: (a) as-deposited, (b) recrystallized.

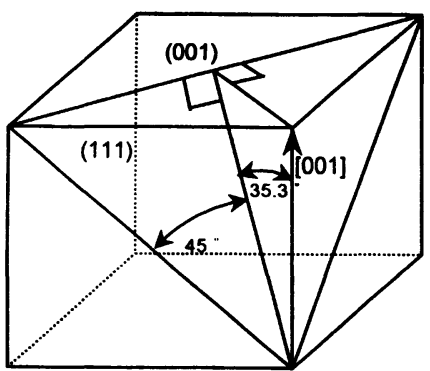

(a)

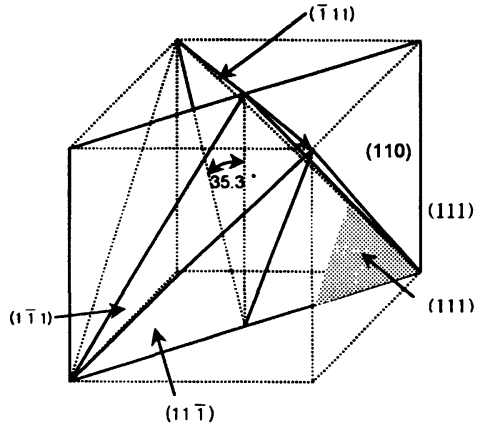

(b)

FIGURE 5 Illustrations of orientation of $\{111\}$ planes in (a) $\langle 100\rangle$ texture, (b) $\langle 110\rangle$ texture. Arrows indicate the directions of growth. 
Figure 6 shows the inverse pole figures of nickel electrodeposit having a $\langle 100\rangle$ texture before and after the recrystallization specimens. The $\langle 100\rangle$ texture did not change even after recrystallization.

Figure 7 shows the inverse pole figures of nickel electrodeposits having a $\langle 110\rangle$ texture before and after the recrystallization specimens. The $\langle 110\rangle$ texture changed to the $\langle\sqrt{3} 10\rangle$ texture after recrystallization.

These results have been explained by the strain energy release maximization model (Lee et al., 1995; Lee, 1996a) as follows.

The driving force for recrystallization in the eletrodeposits is attributed to the stored energy resulting from dislocations. Regardless of the kinds of dislocations, the Burgers vectors of dislocations in the deposits are parallel to the slip directions of the grains. The Burgers vectors in the nickel deposits, therefore, are parallel to the $\langle 110\rangle$ directions.

Lee (1996b) presumed that the stable dislocation array in the electrodeposits would be approximated by edge dislocations, the Burgers vectors of which is nearly normal to the growth direction. This has been experimentally proven (Lee and Lee, 1998).

According to the strain energy release maximization model (Lee et al., 1995; Lee, 1995), the recrystallized grains would orient themselves so that the minimum elastic modulus direction of the recrystallized grains can be parallel to the absolute maximum internal stress direction of the original grains, whereby the strain energy release becomes maximized, because recrystallization takes place in an approximately constant volume system. The absolute maximum internal stress direction in the array of in-plane parallel edge dislocations is parallel to the Burgers vector direction of the dislocations (Lee, 1995). The minimum elastic modulus directions of nickel are the $\langle 100\rangle$ directions. Therefore, the $\langle 110\rangle$ directions of the original nickel grains, which are the Burgers vector directions of nickel will be parallel to the $\langle 100\rangle$ directions of recrystallized grains. There are six equivalent directions in the $\langle 110\rangle$ directions, with opposite directions being taken as the same.

It follows that the $\langle 110\rangle$ directions at right angles or near right angles to the thickness direction of the original deposit become the $\langle 100\rangle$ directions after recrystallization.

For the deposit with the $\langle 100\rangle$ texture, two of the six $\langle 110\rangle$ directions are at right angles, the remaining four are each at an angle of $45^{\circ}$ to the thickness direction, as shown in Fig. 5(a). According to the above 
(a)
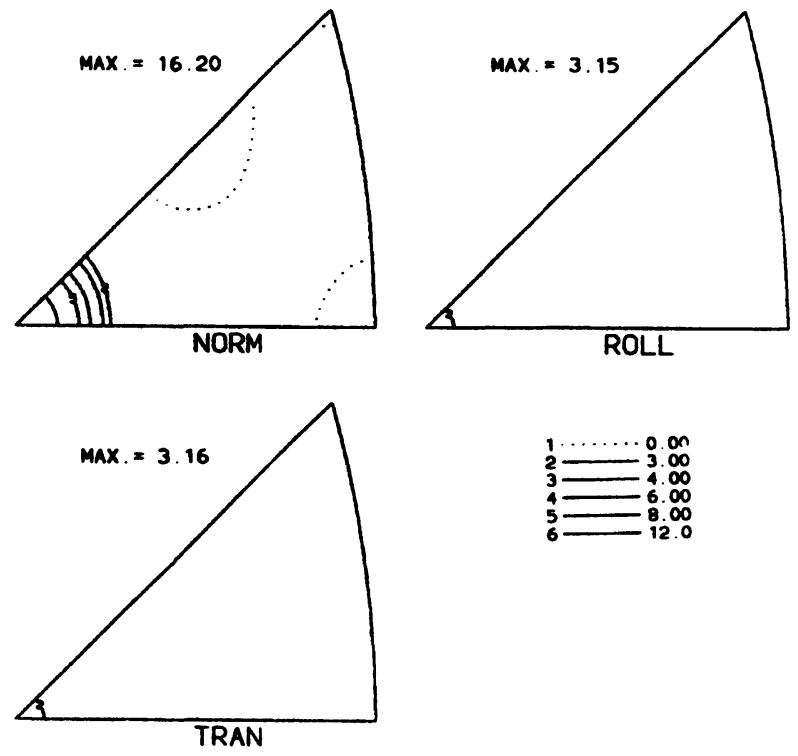

(b)
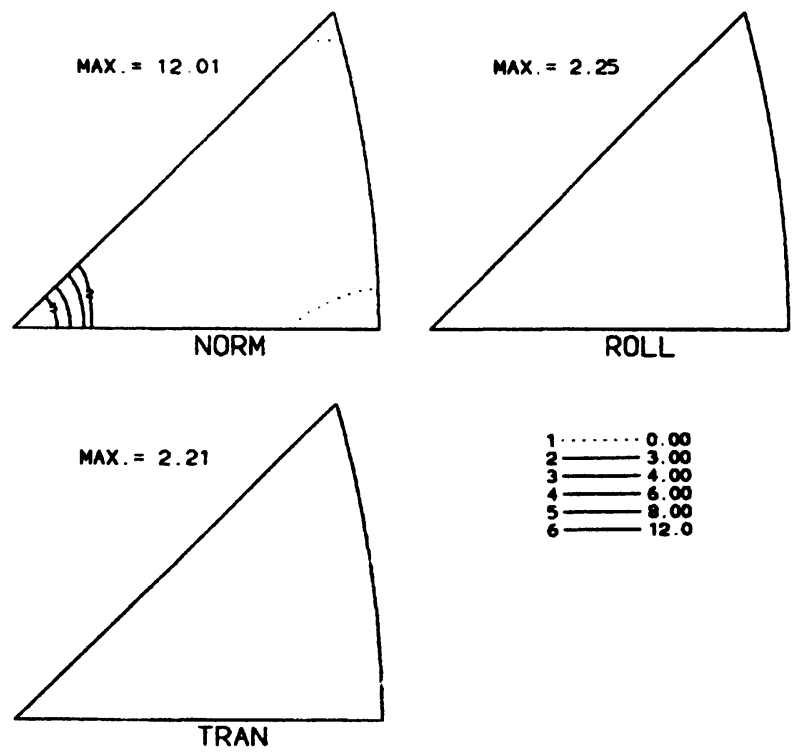

FIGURE 6 The inverse pole figures of nickel electrodeposits having $\langle 100\rangle$ texture: (a) as-deposited, (b) recrystallized. 
(a)
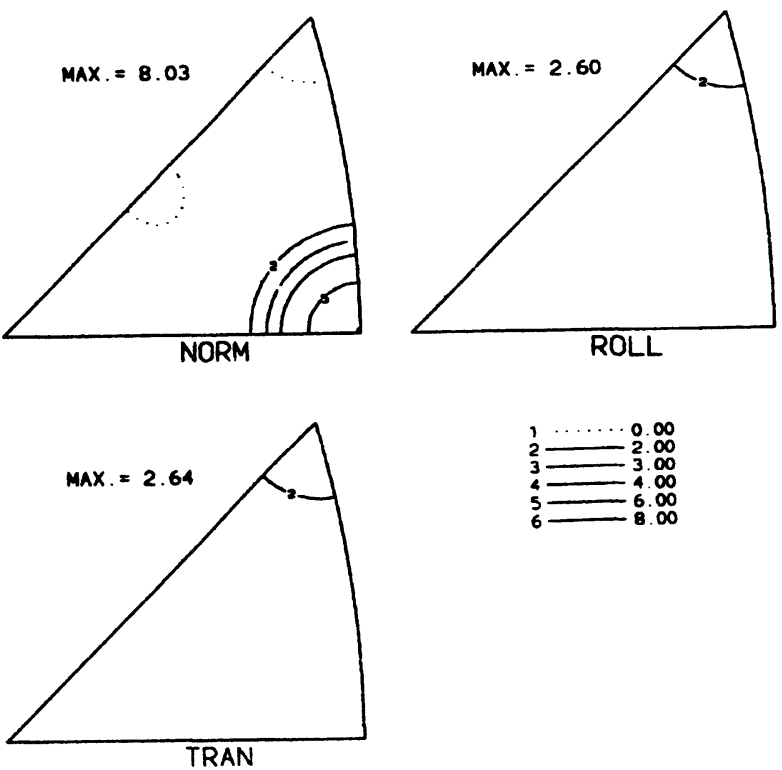

(b)
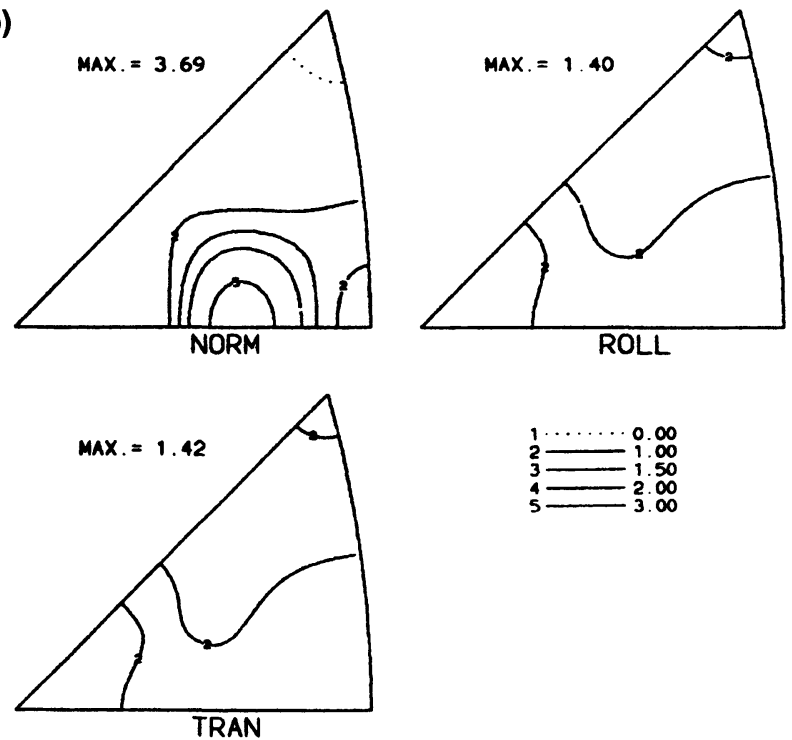

FIGURE 7 The inverse pole figures of nickel electrodeposits having $\langle 110\rangle$ texture: (a) as-deposited, (b) recrystallized. 
model, the former two $\langle 110\rangle$ directions will change to the $\langle 100\rangle$ directions after recrystallization. The recrystallized deposit would then have the $\langle 100\rangle$ texture, as shown in Fig. 5(a), in agreement with the experimental result.

For the $\langle 110\rangle$ texture, one $\langle 110\rangle$ direction is normal to the $\langle 110\rangle$ thickness direction; the remaining four $\langle 110\rangle$ directions are each at angle of $60^{\circ}$ to the $\langle 110\rangle$ thickness direction, as shown in Fig. 5(b). The first one of the $\langle 110\rangle$ and the last four $\langle 110\rangle$ directions are assumed to determine the recrystallization texture because the last four are closer to the planar direction than to the thickness direction. Recalling that the $\langle 110\rangle$ directions change to $\langle 100\rangle$ directions after recrystallization. It follows from Fig. 5(b) that the thickness direction of recrystallized grains should be at angles of $60^{\circ}$ and $90^{\circ}$ with the $\langle 100\rangle$ directions at the same time. The thickness direction satisfying the condition will be $\langle\sqrt{3} 10\rangle$, in agreement with the experimental result.

In summary, the $\langle 100\rangle$ and $\langle 110\rangle$ textures of the nickel electrodeposits obtained from nickel sulfate, nickel chloride and boric acid baths changed to the $\langle 100\rangle$ and $\langle\sqrt{3} 10\rangle$ textures, respectively, when they were recrystallized.

\section{CONCLUSIONS}

(1) The texture of nickel electrodeposits changed from $\langle 100\rangle$ to $\langle 110\rangle$ with increasing the bath temperature.

(2) The $\langle 100\rangle$ and $\langle 110\rangle$ textures of nickel electrodeposits changed to the $\langle 100\rangle$ and $\langle\sqrt{3} 10\rangle$ textures, when recrystallized.

(3) The recrystallization texture of nickel eletrodeposits can be explained by the strain energy release maximization model.

\section{References}

Kang, S., Yang, J. and Lee, D.N. (1995). Relationship between texture and surface morphology of copper electrodeposits. Plating and Surface Finishing, 82(10), 67-70.

Lee, D.N. (1995). The evolution of recrystallization textures from deformation textures. Scripta Metallurigica and Materialia, 32, 1689-1694.

Lee, D.N. (1996a). Texture and related phenomena of copper electrodeposits. Materials Research Society Symposium Proceedings, 427, 167-178.

Lee, D.N. (1996b). Maximum energy release theory for recrystallization textures. Metals and Materials, 2(3), 121-131.

Lee, D.N. and Chang, Y.W. (1974). Changes in preferred orientation and structure of copper deposit depending upon electrolysis conditions. Journal of the Korean Institute of Metals, 12, 243-249. 
Lee, Y.S. and Lee, D.N. (1998). Private communication.

Lee, D.N. and Ye, G.C. (1981). Orientation and microstructure of watts and bright nickel electrodeposits. Plating and Surface Finishing, 68(11), 46-50.

Lee, D.N., Kang, S. and Yang, J. (1995). Relationship between initial and recrystallization textures of copper electrodeposits. Plating and Surface Fininishing, 82(3), 76-79.

Rashkov, S.T., Stoichev, D.S. and Tomov, I. (1972). Influence of current density and temperature on the morphology and preferred orientation of electrodeposited copper coatings. Electrochemica Acta, 17, 1955.

Ye, G.C. and Lee, D.N. (1981). Orientation and microstructure of dull nickel electrodeposits. Plating and Surface Finishing, 68(4), 60.

Ye, X., Bonte, M.D., Celis, J.P. and Roos, J.R. (1992). Role of overpotential on texture, morphology and ductility of electrodeposited copper foils for printed circuit board applications. Journal of Electrochem Soc., 139, 1592. 